\title{
Two-Stage Multiobjective Optimization for Emergency Supplies Allocation Problem under Integrated Uncertainty
}

\author{
Xuejie Bai ${ }^{1,2}$ \\ ${ }^{1}$ College of Science, Hebei Agricultural University, Baoding, Hebei 071001, China \\ ${ }^{2}$ College of Management, Hebei University, Baoding, Hebei 071002, China \\ Correspondence should be addressed to Xuejie Bai; hebaubxj@163.com
}

Received 15 February 2016; Revised 11 April 2016; Accepted 11 April 2016

Academic Editor: Shuming Wang

Copyright (c) 2016 Xuejie Bai. This is an open access article distributed under the Creative Commons Attribution License, which permits unrestricted use, distribution, and reproduction in any medium, provided the original work is properly cited.

\begin{abstract}
This paper proposes a new two-stage optimization method for emergency supplies allocation problem with multisupplier, multiaffected area, multirelief, and multivehicle. The triplet of supply, demand, and the availability of path is unknown prior to the extraordinary event and is descriptive with fuzzy random variable. Considering the fairness, timeliness, and economical efficiency, a multiobjective expected value model is built for facility location, vehicle routing, and supply allocation decisions. The goals of proposed model aim to minimize the proportion of demand nonsatisfied and response time of emergency reliefs and the total cost of the whole process. When the demand and the availability of path are discrete, the expected values in the objective functions are converted into their equivalent forms. When the supply amount is continuous, the equilibrium chance in the constraint is transformed to its equivalent one. To overcome the computational difficulty caused by multiple objectives, a goal programming model is formulated to obtain a compromise solution. Finally, an example is presented to illustrate the validity of the proposed model and the effectiveness of the solution method.
\end{abstract}

\section{Introduction}

Disasters mainly include both natural disasters such as earthquakes, floods, hurricanes, and man-made disasters like terrorist attacks or chemical leakages. When a disaster strikes, much infrastructure lacking adequate structural stability collapses and human life either die or need immediate help. As an important role in disaster management, emergency logistics management has aroused increasing research interests from both logistics academics and practitioners in recent years. Altay and Green III [1] and Galindo and Batta [2] showed that disaster management has gained importance in the last two decades in both Operations Research and Management Science research. Abounacer et al. [3] presented a three-objective location-transportation model for disaster response and adopted an epsilon-constraint method for generating the exact Pareto frontier. Barzinpour and Esmaeili [4] developed a multiobjective relief chain location distribution model for preparation planning phase of disaster management which was applied to a real case study of an urban district in Iran. For the latest development of emergency logistics and disaster operations management, the interested readers may refer to Anaya-Arenas et al. [5], Hoyos et al. [6], Özdamar and Ertem [7], and Zheng et al. [8].

There exists much uncertainty about whether or not the disasters will happen, and if they do, where and with what magnitude. Uncertainty is a paramount challenge when creating an efficient and effective emergency plan. As pointed by Bozorgi-Amiri et al. [9], information about demand, supply, and cost is unknown in advance due to the lack of related data. The existence of uncertainty has motivated some researchers to address the critical parameters in the form of stochastic approaches. For example, Salmerón and Apte [10] developed a two-stage stochastic optimization model for natural disaster asset prepositioning problem under the uncertainty of the event's location and severity. Rawls and Turnquist [11] presented a two-stage stochastic mixed-integer programming model for prepositioning disaster relief purposes, which was solved by a heuristic solution approach called the Lagrangian L-shaped method intended for large-scale problem instances. In preparation for the sudden-onset disasters, Balcik and $\mathrm{Ak}$ [12] studied the supplier selection problem of a relief 
organization and developed a stochastic programming model by using a scenario-based approach to represent demand uncertainty. Zhang and Jiang [13] employed a robust counterpart approach to study the biobjective emergency medical services design problem under uncertainty and found model's Pareto-optimal solutions for costs and response times by the weighting method.

Since the development of fuzzy optimization techniques, fuzzy theory has been developed for emergency supplies allocation problem to model uncertainties mathematically. For example, Sheu [14] presented a hybrid fuzzy clusteringoptimization approach to a three-layer emergency logistics codistribution conceptual framework and demonstrated the applicability of the new method and potential advantages through numerical studies. Sun et al. [15] built an emergency material demand prediction model by integrating the fuzzy set and rough set theory and developed decision rules and computing methods based on the risk decision-making principle of classical operational research. Zheng and Ling [16] proposed a multiobjective fuzzy emergency transportation planning model for the disaster relief supply chains and designed a cooperative optimization method to efficiently solve the proposed problem. Bai [17] addressed a fuzzy prepositioning problem for emergency supplies on the basis of fuzzy possibility theory [18], by which the transportation costs, the suppliers' supply amounts, the affected areas' demands, and the capacities of the roads were characterized by type-2 fuzzy variables. For other applications of fuzzy possibility theory, please refer to Bai and Liu [19] and references therein.

In practical emergency supplies allocation problem, decision-makers may encounter an integrated uncertain environment where randomness and fuzziness often coexist when predicting the impact resulting from the extraordinary event and arising from the vulnerability of the transportation system. To handle the twofold uncertainty, it is a suitable choice to employ fuzzy random theory. The notion of fuzzy random variable was first introduced by Kwakernaak [20] and further refined by the work of Y.-K. Liu and B. Liu [21]. Based on fuzzy random variables, several kinds of fuzzy-stochastic optimization models have been proposed in recent years. For example, Ben Abdelaziz and Masri [22] presented the multistage stochastic program with fuzzy probability distribution and discussed its solution strategy. Wang and Watada [23] introduced the two-stage fuzzy stochastic model with value-at-risk criterion. Wang and Pedrycz [24] developed two kinds of robust granular optimization models under hybrid uncertainty for tackling the heavy computational burden. What is more, fuzzy stochastic programming has been successfully applied in some real world optimization problems. Li et al. [25] employed the multistage fuzzystochastic programming model for helping decision-maker to identify the water-resources allocation and management policies under uncertainty. Wang et al. [26] studied twostage fuzzy random facility location model with recourse where both the demands and costs were assumed to be fuzzy random variables. Different from the viewpoint of risk neutral criterion [26], Wang and Watada [27] built a valueat-risk based fuzzy random facility location model. Yuan [28] proposed a two-stage fuzzy optimization method for solving the multiproduct multiperiod production planning problem. Wang et al. [29] used robust optimization with information granules for distributional parameters to develop a two-stage waste-to-energy feedstock flow planning model. This paper differs from the above-mentioned work in following aspect. We explore the potential of equilibrium optimization [30] to manage fuzziness and randomness for complex emergency supplies allocation problem. For the recent development of equilibrium optimization, we refer the reader to [31, 32] for detailed discussion.

The aim of this paper is to extend the stochastic or fuzzy methods mentioned above and adopt equilibrium optimization method to address the emergency supplies allocation problem. To the best of our knowledge, the emergency supplies allocation problem under integrated uncertainty has not yet been studied in the existing literature. The main contributions of the paper are summarized as follows. (1) This paper applies equilibrium optimization to examine the impact of uncertain demands of the affected areas, the availability of path, and supply amounts on the optimal allocation plan. The demands of the affected areas, the availability of path, and supply amounts are unknown prior to the event and are characterized by fuzzy random variables. (2) This paper develops a two-stage emergency supplies allocation model with expected objective functions and equilibrium chance constraints. The supplier location decision is made before the disaster occurs, which is the first-stage decision. Once disaster happens, the decision-maker needs to determine the second-stage decisions of vehicle routing and reliefs allocation as quickly as possible. (3) The solution framework incorporates the equivalent representations by considering the uncertain parameters with appropriate possibility and probability distributions and goal programming formulation, which makes the original emergency supplies allocation model computationally tractable. Numerical example in the context of emergency logistics is provided to demonstrate the value of two-stage equilibrium model and the efficiency of the presented method.

The rest of this paper is organized as follows. In Section 2 we formulate a fuzzy random emergency supplies allocation problem. In Section 3 we convert the original uncertain emergency supplies allocation model to an equivalent deterministic mixed-integer problem and solve it by the goal programming method in Section 4. We study an example of the emergency supplies of an earthquake by using the fuzzy random model established in this paper and the validity of the methods is also tested in this example in Section 5. Finally, Section 6 gives the conclusions.

\section{Two-Stage Emergency Supplies Allocation Model}

This paper addresses a new two-stage optimization method for emergency supplies allocation problem with multisupplier, multiaffected area, multirelief, and multivehicle. The emergency logistics network is composed of two primary members: relief suppliers and affected areas. The decision 
process in the emergency supplies allocation problem is divided into two stages. The first stage considers the selection of a set of candidate relief suppliers and the amount of various vehicles, whereas the second stage deals with the decisions in terms of vehicle routing and reliefs allocation in every disaster scenario.

For the sake of simplicity, we adopt the notations displayed as follows.

Indices. Consider

$I$ : set of potential relief suppliers, indexed by $i$;

$J$ : set of affected areas, indexed by $j$;

$K$ : set of reliefs, indexed by $k$;

$M$ : set of vehicle types, indexed by $m$;

$\Omega \times \Gamma$ : set of disaster scenarios, indexed by $(\omega, \gamma)$.

\section{Fixed Parameters. Consider}

$T$ : threshold of coverage;

$a_{m}$ : unit travel time of vehicle type $m$;

$b_{m}$ : unit travel cost of vehicle type $m$;

$c_{i m}$ : unit fixed cost of vehicle type $m$ in relief supplier $i$

$g_{i}:$ unit fixed cost of the relief supplier $i$;

$V_{m}$ : load capacity of vehicle type $m$;

$e_{k}$ : average weight of relief $k$;

$t_{i j}$ : distance from relief supplier $i$ to affected area $j$;

$U_{i m}$ : capacity of vehicle type $m$ in relief supplier $i$;

$\alpha, \beta$ : confidence level in unit interval $[0,1]$.

Fuzzy Random Variables. Consider

$\xi_{i k}$ : maximum supply amount of relief $k$ in relief supplier $i$;

$d_{j k}$ : amount of demand for commodity $k$ in affected area $j$;

$\zeta_{i j}$ : availability of path from relief supplier $i$ to affected area $j$;

$\eta_{i j}$ : available distance from relief supplier $i$ to affected area $j$.

\section{Decision Variables. Consider}

$w_{i}$ : a binary variable indicating whether a relief supplier $i$ is chosen or not;

$x_{i m}$ : an integer number of vehicle type $m$ gathered in relief supplier $i$;

$y_{i j m}$ : an integer number of vehicle type $m$ assigned to the path from relief supplier $i$ to affected area $j$;

$z_{i j k m}$ : amount of relief $k$ shipped from relief supplier $i$ to affected area $j$ by vehicle type $m$.
On the basis of the notations above, we formally build a fuzzy random emergency supplies allocation model with respect to disaster management as follows:

$$
\begin{aligned}
\min _{1}= & \frac{\left(f_{1}, f_{2}, f_{3}\right)}{E\left[\sum_{j \in J} \sum_{k \in K}\left(d_{j k}-\sum_{i \in I} \sum_{m \in M} z_{i j k m}\right)\right]} \\
f_{2}\left[\sum_{j \in J} \sum_{k \in K} d_{j k}\right] & E\left[\sum_{i \in I} \sum_{j \in J} \sum_{m \in M} a_{m} \eta_{i j} y_{i j m}\right] \\
f_{3} & {\left[\sum_{i \in I} g_{i} w_{i}+\sum_{i \in I} \sum_{m \in M} c_{i m} x_{i m}+\sum_{i \in I} \sum_{j \in J} \sum_{m \in M} b_{m} \eta_{i j} y_{i j m}\right] }
\end{aligned}
$$

$\forall i \in I, k \in K$

$\sum_{i \in I} \sum_{m \in M} z_{i j k m} \leq d_{j k}, \quad \forall j \in J, k \in K$

$\eta_{i j}=\left\{\begin{array}{ll}t_{i j}, & \text { if } \zeta_{i j}=1 \\ +\infty, & \text { if } \zeta_{i j}=0,\end{array} \quad \forall i \in I, j \in J\right.$

$y_{i j m}\left\{\begin{array}{ll}\geq 0, & \text { if } \eta_{i j} \leq T \\ =0, & \text { if } \eta_{i j}>T,\end{array} \quad \forall i \in I, \quad j \in J, m \in M\right.$

$w_{i} \in\{0,1\}, \quad \forall i \in I$

$x_{i m} \geq 0$, integer, $\forall i \in I, m \in M$

$y_{i j m} \geq 0$, integer, $\forall i \in I, \quad j \in J, m \in M$

$z_{i j k m} \geq 0, \quad \forall i \in I, \quad j \in J, m \in M, k \in K$.

Equation (1) shows that the method selected is a multiobjective optimization model for emergency supplies allocation problem with multisupplier, multiaffected area, multirelief, and multivehicle emergency logistics network. The first objective function (2) is to minimize the expected proportion of unmet demands and represents "fairness" in process of relief resources allocation. The second objective function (3) of the proposed model is to minimize the expected total travel time of relief allocation and stands for "timeliness" of relief resources allocation. The third objective function (4) is to minimize the total cost and implies the "economy." 
Constraint (5) defines the capacity limits of vehicle in relief supplier. If a relief supplier is made available at location $i$, various vehicles can be gathered there. Constraint (6) requires that the amount of vehicle at work cannot exceed the supplier's real capacity. Constraint (7) imposes the load capacity limits of vehicle and ensures the existence of commodity flow. Constraint (8) means that the allocation amount of commodity should not exceed the amount of supply. It is expressed as a chance constraint to handle the uncertainty of supply of relief resources with the predefined confidence levels, usually close to 1 . Constraint (9) implies the relationship between allocation amount and demand, which enforces that allocation amount should not exceed the amount of demand. Constraint (10) ensures the availability of path. Uncertainty resulting from the vulnerability of the transportation system leads to unavailability of some paths. $\zeta_{i j}$ equals 1 if the path is unobstructed and 0 otherwise. The available distance $\eta_{i j}$ will be infinite when the path is destroyed. Constraint (11) emphasizes the coverage limits. Finally, constraints (12), (13), (14), and (15) ensure the nonnegativity and integer of decision variables.

Remark 1. The reasons that the equilibrium chance measure is used in supply capacity constraint (8) of emergency supplies allocation model are as follows. Firstly, the maximum supply amount $\xi_{i k}$ of relief $k$ in relief supplier $i$ is organized before the disaster happens, so it is inappropriate to represent supply by a set of discrete scenarios. We suppose that the supply amount is characterized by fuzzy random variable with continuous possibility and probability distribution functions. The equilibrium measure is employed to gauge the chance of a fuzzy random event with twofold uncertainty. Secondly, the equilibrium chance $\mathrm{Ch}$ of a fuzzy random event is defined as

$$
\begin{aligned}
\operatorname{Ch} & \{f(\xi) \leq 0\} \\
& =\sup _{(\alpha, \beta) \in[0,1]^{2}}\{\alpha \wedge \beta \mid \operatorname{Pr}\{\operatorname{Cr}\{f(\xi) \leq 0\} \geq \alpha\} \geq \beta\} .
\end{aligned}
$$

According to the property of equilibrium chance, the supply capacity constraint (8) is equivalent to the following form:

$$
\mathrm{Ch}\left\{\sum_{j \in J} \sum_{m \in M} z_{i j k m} \leq \xi_{i k}\right\} \geq \alpha \wedge \beta, \quad \forall i \in I, k \in K .
$$

Obviously, the current representation (8) is easier to turn a computationally tractable problem. Thirdly, if fuzzy random supply amount reduces to fuzzy variable, the chance constraint (8) becomes the following credibility constraint:

$$
\operatorname{Cr}\left\{\sum_{j \in J} \sum_{m \in M} z_{i j k m} \leq \xi_{i k}\right\} \geq \alpha, \quad \forall i \in I, k \in K .
$$

If fuzzy random supply amount reduces to random variable, the chance constraint (8) becomes the following probability constraint:

$$
\operatorname{Pr}\left\{\sum_{j \in J} \sum_{m \in M} z_{i j k m} \leq \xi_{i k}\right\} \geq \beta, \quad \forall i \in I, k \in K .
$$

So the equilibrium chance measure extends both the credibility measure in fuzzy environment and the probability measure in stochastic environment, which is an ideal tool under integrated uncertainty.

\section{Model Analysis}

3.1. Handling Expectation Objective Functions. We first consider the analytical expression of the fairness, timeliness, and total cost objective functions (2)-(4); that is,

$$
\begin{aligned}
f_{1} & =\frac{E\left[\sum_{j \in J} \sum_{k \in K}\left(d_{j k}-\sum_{i \in I} \sum_{m \in M} z_{i j k m}\right)\right]}{E\left[\sum_{j \in J} \sum_{k \in K} d_{j k}\right]}, \\
f_{2} & =E\left[\sum_{i \in I} \sum_{j \in J} \sum_{m \in M} a_{m} \eta_{i j} y_{i j m}\right], \\
f_{3} & =E\left[\sum_{i \in I} g_{i} w_{i}+\sum_{i \in I} \sum_{m \in M} c_{i m} x_{i m}\right. \\
& \left.+\sum_{i \in I} \sum_{j \in J} \sum_{m \in M} b_{m} \eta_{i j} y_{i j m}\right] .
\end{aligned}
$$

Theorem 2. Consider the fairness, timeliness, and total cost objective functions (2), (3), and (4) in emergency supplies allocation problem (1)-(15). Suppose that the disaster scenario is a discrete fuzzy random variable. For any $\omega_{s}$, the corresponding scenario is a normalized discrete fuzzy variable with the following possibility distribution:

$$
\mu(\gamma)= \begin{cases}\mu_{1}, & \text { if } \gamma=\gamma_{1} \\ \mu_{2}, & \text { if } \gamma=\gamma_{2} \\ \vdots & \\ \mu_{N}, & \text { if } \gamma=\gamma_{N},\end{cases}
$$

where $\mu_{n}=\operatorname{Pos}\left\{\gamma=\gamma_{n}\right\}>0$ and $\max _{n=1}^{N} \mu_{n}=1$. Let the probability of $\omega_{s}$ is $p_{s}$, and $\sum_{s=1}^{S} p_{s}=1$.

(i) The fairness objective function (2) is equivalent to

$$
\begin{aligned}
& f_{1} \\
& =\frac{\sum_{s \in S} \sum_{n \in N} \sum_{j \in J} \sum_{k \in K} p_{s} q_{n}\left(d_{j k}^{s n}-\sum_{i \in I} \sum_{m \in M} z_{i j k m}^{s n}\right)}{\sum_{s \in S} \sum_{n \in N} \sum_{j \in J} \sum_{k \in K} p_{s} q_{n} d_{j k}^{s n}} .
\end{aligned}
$$

(ii) The timeliness objective function (3) is equivalent to

$$
f_{2}=\sum_{s \in S} \sum_{n \in N} \sum_{i \in I} \sum_{j \in J} \sum_{m \in M} p_{s} q_{n} a_{m} \eta_{i j}^{s n} y_{i j m}^{s n} .
$$

(iii) The total cost objective function (4) is equivalent to

$$
\begin{aligned}
f_{3}= & \sum_{i \in I} g_{i} w_{i}+\sum_{i \in I} \sum_{m \in M} c_{i m} x_{i m} \\
& +\sum_{s \in S} \sum_{n \in N} \sum_{i \in I} \sum_{j \in J} \sum_{m \in M} p_{s} q_{n} b_{m} \eta_{i j}^{s n} y_{i j m}^{s n},
\end{aligned}
$$


where

$$
\begin{aligned}
q_{n}= & \frac{1}{2}\left(\max _{h=1}^{n} \mu_{h}-\max _{h=0}^{n-1} \mu_{h}\right) \\
& +\frac{1}{2}\left(\max _{h=n}^{N} \mu_{h}-\underset{h=n+1}{N+1} \mu_{h}\right),
\end{aligned}
$$

and $\mu_{0}=\mu_{N+1}=0$.

Proof. We only prove assertion (i), and assertions (ii)-(iii) can be proved similarly.

Since the disaster scenario is a discrete fuzzy random variable, for any $\omega_{s}$, the corresponding scenario is a normalized discrete fuzzy variable with the following possibility distribution:

$$
\mu(\gamma)= \begin{cases}\mu_{1}, & \text { if } \gamma=\gamma_{1} \\ \mu_{2}, & \text { if } \gamma=\gamma_{2} \\ \vdots & \\ \mu_{N}, & \text { if } \gamma=\gamma_{N},\end{cases}
$$

where $\mu_{n}=\operatorname{Pos}\left\{\gamma=\gamma_{n}\right\}>0$ and $\max _{n=1}^{N} \mu_{n}=1$. Without loss of generality, suppose that $d_{j k, \omega_{s}}\left(\gamma_{n}\right), n=1,2, \ldots, N$, satisfy $d_{j k, \omega_{s}}\left(\gamma_{1}\right) \leq d_{j k, \omega_{s}}\left(\gamma_{2}\right) \leq \cdots \leq d_{j k, \omega_{s}}\left(\gamma_{N}\right)$; then the expected value of fuzzy variable $d_{j k, \omega_{s}}$ is

$$
E_{\gamma}\left[d_{j k, \omega_{s}}\right]=\sum_{n \in N} q_{n} d_{j k, \omega_{s}}\left(\gamma_{n}\right)
$$

where

$$
\begin{aligned}
q_{n}= & \frac{1}{2}\left(\max _{h=1}^{n} \mu_{h}-\max _{h=0}^{n-1} \mu_{h}\right) \\
& +\frac{1}{2}\left(\max _{h=n}^{N} \mu_{h}-\max _{h=n+1}^{N+1} \mu_{h}\right),
\end{aligned}
$$

and $\mu_{0}=\mu_{N+1}=0$. It is known that $q_{n} \geq 0$ and $\sum_{n=1}^{N} q_{n}=$ $\max _{n=1}^{N} \mu_{n}=1$. have

Noting that the probability of $\omega_{s}$ is $p_{s}$ and $\sum_{s=1}^{S} p_{s}=1$, we

$$
\begin{aligned}
E\left[d_{j k}\right] & =E_{\omega} E_{\gamma}\left[d_{j k, \omega_{s}}\right]=\sum_{s \in S} \sum_{n \in N} p_{s} q_{n} d_{j k, \omega_{s}}\left(\gamma_{n}\right) \\
& =\sum_{s \in S} \sum_{n \in N} p_{s} q_{n} d_{j k}^{s n} .
\end{aligned}
$$

Therefore,

$$
\begin{aligned}
& f_{1}=\frac{E\left[\sum_{j \in J} \sum_{k \in K}\left(d_{j k}-\sum_{i \in I} \sum_{m \in M} z_{i j k m}\right)\right]}{E\left[\sum_{j \in J} \sum_{k \in K} d_{j k}\right]} \\
& =\frac{\sum_{s \in S} \sum_{n \in N} \sum_{j \in J} \sum_{k \in K} p_{s} q_{n}\left(d_{j k}^{s n}-\sum_{i \in I} \sum_{m \in M} z_{i j k m}^{s n}\right)}{\sum_{s \in S} \sum_{n \in N} \sum_{j \in J} \sum_{k \in K} p_{s} q_{n} d_{j k}^{s n}} .
\end{aligned}
$$

The proof of assertion (i) is complete.
3.2. Dealing with Equilibrium Constraint. In the following, we deal with the analytical expressions of supply capacity chance constraint (8); that is,

$$
\operatorname{Pr}\left\{\operatorname{Cr}\left\{\sum_{j \in J} \sum_{m \in M} z_{i j k m} \leq \xi_{i k}\right\} \geq \alpha\right\} \geq \beta,
$$

$\forall i \in I, k \in K$.

Lemma 3. Consider the supply capacity chance constraint (8) in emergency supplies allocation problem (1)-(15). Let the supply amount $\xi_{i k}$ be a triangular fuzzy random variable. For any $\omega, \xi_{i k, \omega}=\left(X_{i k, \omega}-a_{i k}, X_{i k, \omega}, X_{i k, \omega}+b_{i k}\right)$, where $a_{i k}, b_{i k} \geq 0$, and $X_{i k}$ is a random variable.

(i) If $0<\alpha<1 / 2$, then the supply capacity chance constraint (8) is equivalent to

$$
\operatorname{Pr}\left\{\sum_{j \in J} \sum_{m \in M} z_{i j k m} \leq X_{i k, \omega}+(1-2 \alpha) b_{i k}\right\} \geq \beta \text {, }
$$

$\forall i \in I, k \in K$.

(ii) If $1 / 2 \leq \alpha \leq 1$, then the supply capacity chance constraint (8) is equivalent to

$\operatorname{Pr}\left\{\sum_{j \in J} \sum_{m \in M} z_{i j k m} \leq X_{i k, \omega}-(2 \alpha-1) a_{i k}\right\} \geq \beta$,

$\forall i \in I, k \in K$.

Proof. We only prove assertion (i), and assertion (ii) can be proved similarly.

Since $\xi_{i k}(\omega)=\left(X_{i k, \omega}-a_{i k}, X_{i k, \omega}, X_{i k, \omega}+b_{i k}\right)$, its possibility distribution is given as follows:

$$
\begin{aligned}
v_{\xi_{i k}(\omega)}(x) & \begin{cases}\frac{x-X_{i k, \omega}+a_{i k}}{a_{i k}}, & x \in\left[X_{i k, \omega}-a_{i k}, X_{i k, \omega}\right] \\
\frac{X_{i k, \omega}+b_{i k}-x}{b_{i k}}, & x \in\left[X_{i k, \omega}, X_{i k, \omega}+b_{i k}\right] \\
0 . & \end{cases}
\end{aligned}
$$

When $0<\alpha<1 / 2$, then we have

$$
\begin{aligned}
\operatorname{Cr} & \left\{\sum_{j \in J} \sum_{m \in M} z_{i j k m} \leq \xi_{i k}\right\} \\
& =\frac{1}{2}\left(1+\sup _{x \geq t} \nu_{\xi_{i k, \omega}}(x)-\sup _{x<t} \nu_{\xi_{i k, \omega}}(x)\right) \\
& =\frac{1}{2} \sup _{x \geq t} v_{\xi_{i k, \omega}}(x),
\end{aligned}
$$

where $t=\sum_{j \in J} \sum_{m \in M} z_{i j k m}$. Thus $\operatorname{Cr}\left\{\sum_{j \in J} \sum_{m \in M} z_{i j k m} \leq\right.$ $\left.\xi_{i k}\right\} \geq \alpha$ is equivalent to

$$
\sup _{x \geq t} \nu_{\xi_{i k, \omega}}(x) \geq 2 \alpha \text {. }
$$


If we denote

$$
\left(\xi_{i k, \omega}\right)_{\text {sup }}(\delta)=\sup \left\{t \mid \sup _{x \geq t} \nu_{\xi_{i k, \omega}}(x) \geq \delta\right\}
$$

for $\delta \in(0,1]$, then $\left(\xi_{i k}(\omega)\right)_{\text {sup }}(2 \alpha) \geq t$. Therefore, the following inequity

$$
\left(\xi_{i k, \omega}\right)_{\text {sup }}(2 \alpha) \geq \sum_{j \in J} \sum_{m \in M} z_{i j k m}
$$

holds.

According to the possibility distribution of $\nu_{\xi_{i k, \omega}}(x)$, $\left(\xi_{i k, \omega}\right)_{\text {sup }}(2 \alpha)$ is the solution of the following equation:

$$
\frac{X_{i k, \omega}+b_{i k}-x}{b_{i k}}-2 \alpha=0 \text {. }
$$

Solving the above equation, we have

$$
\left(\xi_{i k, \omega}\right)_{\text {sup }}(2 \alpha)=X_{i k, \omega}+(1-2 \alpha) b_{i k} .
$$

So $\operatorname{Cr}\left\{\sum_{j \in J} \sum_{m \in M} z_{i j k m} \leq \xi_{i k}\right\} \geq \alpha$ is equivalent to

$$
X_{i k, \omega}+(1-2 \alpha) b_{i k} \geq \sum_{j \in J} \sum_{m \in M} z_{i j k m} .
$$

Therefore, the supply capacity chance constraint (8) is equivalent to

$$
\operatorname{Pr}\left\{\sum_{j \in J} \sum_{m \in M} z_{i j k m} \leq X_{i k, \omega}+(1-2 \alpha) b_{i k}\right\} \geq \beta,
$$

$\forall i \in I, k \in K$.

The proof of assertion $(i)$ is complete.

Based on Lemma 3, when $X_{i k} \sim \mathcal{N}\left(\mu_{i k}, \sigma_{i k}^{2}\right)$, we can have the following result.

Theorem 4. Consider the supply capacity chance constraint (8) in emergency supplies allocation problem (1)-(15). Let the supply amount $\xi_{i k}$ be a triangular fuzzy random variable. For any $\omega, \xi_{i k, \omega}=\left(X_{i k, \omega}-a_{i k}, X_{i k, \omega}, X_{i k, \omega}+b_{i k}\right)$, where $a_{i k}, b_{i k} \geq 0$ and $X_{i k} \sim \mathcal{N}\left(\mu_{i k}, \sigma_{i k}^{2}\right)$.

(i) If $0<\beta<1 / 2$, then the supply capacity chance constraint (8) is equivalent to

$$
\sum_{j \in J} \sum_{m \in M} z_{i j k m} \leq \mu_{i k}+\sigma_{i k} \Phi^{-1}(1-\beta)+(1-2 \alpha) b_{i k},
$$

$\forall i \in I, k \in K$.

(ii) If $1 / 2 \leq \beta \leq 1$, then the supply capacity chance constraint (8) is equivalent to

$\sum_{j \in J} \sum_{m \in M} z_{i j k m} \leq \mu_{i k}+\sigma_{i k} \Phi^{-1}(1-\beta)-(2 \alpha-1) a_{i k}$

$\forall i \in I, k \in K$, where $\Phi(\cdot)$ is the cumulative distribution function of standard normal random variable.

Proof. We only prove assertion (i), and assertion (ii) can be proved similarly.

From Lemma 3, it is known that the supply capacity chance constraint (8) is equivalent to

$$
\operatorname{Pr}\left\{\sum_{j \in J} \sum_{m \in M} z_{i j k m}-(1-2 \alpha) b_{i k} \leq X_{i k}\right\} \geq \beta,
$$

$\forall i \in I, k \in K$.

Since $X_{i k} \sim \mathcal{N}\left(\mu_{i k}, \sigma_{i k}^{2}\right)$, we have

$$
\frac{X_{i k}-\mu_{i k}}{\sigma_{i k}} \sim \mathcal{N}(0,1) .
$$

So

$$
\begin{aligned}
\operatorname{Pr} & \left\{\sum_{j \in J} \sum_{m \in M} z_{i j k m}-(1-2 \alpha) b_{i k} \leq X_{i k}\right\} \\
& =\operatorname{Pr}\left\{\frac{\sum_{j \in J} \sum_{m \in M} z_{i j k m}-(1-2 \alpha) b_{i k}-\mu_{i k}}{\sigma_{i k}}\right. \\
& \left.\leq \frac{X_{i k}-\mu_{i k}}{\sigma_{i k}}\right\}=1 \\
& -\Phi\left(\frac{\sum_{j \in J} \sum_{m \in M} z_{i j k m}-(1-2 \alpha) b_{i k}-\mu_{i k}}{\sigma_{i k}}\right) .
\end{aligned}
$$

Therefore, probability constraint (45) is equivalent to

$$
\begin{array}{r}
\Phi\left(\frac{\sum_{j \in J} \sum_{m \in M} z_{i j k m}-(1-2 \alpha) b_{i k}-\mu_{i k}}{\sigma_{i k}}\right) \leq 1-\beta, \\
\forall i \in I, \quad k \in K .
\end{array}
$$

That is to say,

$$
\sum_{j \in J} \sum_{m \in M} z_{i j k m} \leq \mu_{i k}+\sigma_{i k} \Phi^{-1}(1-\beta)+(1-2 \alpha) b_{i k}
$$

$\forall i \in I, k \in K$.

The proof of assertion (i) is complete.

3.3. Equivalent Mixed-Integer Model. In view of the discussion above, when the uncertain variables are mutually independent, the exact analytical expressions of the objectives and constraints are available. Finally, we can reformulate the 
equivalent model of emergency supplies allocation problem in the subsequent form:

$$
\begin{aligned}
& \min \left(f_{1}, f_{2}, f_{3}\right) \\
& f_{1} \\
& =\frac{\sum_{s \in S} \sum_{n \in N} \sum_{j \in J} \sum_{k \in K} p_{s} q_{n}\left(d_{j k}^{s n}-\sum_{i \in I} \sum_{m \in M} z_{i j k m}^{s n}\right)}{\sum_{s \in S} \sum_{n \in N} \sum_{j \in J} \sum_{k \in K} p_{s} q_{n} d_{j k}^{s n}} \\
& f_{2}=\sum_{s \in S} \sum_{n \in N} \sum_{i \in I} \sum_{j \in J} \sum_{m \in M} p_{s} q_{n} a_{m} \eta_{i j}^{s n} y_{i j m}^{s n} \\
& f_{3} \\
& =\sum_{i \in I} g_{i} w_{i}+\sum_{i \in I} \sum_{m \in M} c_{i m} x_{i m} \\
& +\sum_{s \in S} \sum_{n \in N} \sum_{i \in I} \sum_{j \in J} \sum_{m \in M} p_{s} q_{n} b_{m} \eta_{i j}^{s n} y_{i j m}^{s n} \\
& \text { s.t. } \quad x_{i m} \leq U_{i m} w_{i}, \quad \forall i \in I, m \in M \\
& \sum_{j \in J} y_{i j m}^{s n} \leq x_{i m}, \quad \forall i \in I, m \in M, s \in S, n \in N \\
& \sum_{k \in K} e_{k} z_{i j k m}^{s n} \leq V_{m} y_{i j m}^{s n}, \\
& \forall i \in I, \quad j \in J, m \in M, s \in S, n \in N \\
& \sum_{j \in J} \sum_{m \in M} z_{i j k m}^{s n} \leq \mu_{i k}+\sigma_{i k} \Phi^{-1}(1-\beta)-(2 \alpha-1) a_{i k}, \\
& \forall i \in I, k \in K, s \in S, n \in N \\
& \sum_{i \in I} \sum_{m \in M} z_{i j k m}^{s n} \leq d_{j k}^{s n}, \quad \forall j \in J, k \in K, s \in S, n \in N \\
& \eta_{i j}^{s n}=\left\{\begin{array}{ll}
t_{i j}, & \text { if } \zeta_{i j}^{s n}=1 \\
+\infty, & \text { if } \zeta_{i j}^{s n}=0,
\end{array} \quad \forall i \in I, j \in J, s \in S, n \in N\right. \\
& y_{i j m}^{s n} \begin{cases}\geq 0, & \text { if } \eta_{i j}^{s n} \leq T \\
=0, & \text { if } \eta_{i j}^{s n}>T,\end{cases} \\
& \forall i \in I, \quad j \in J, m \in M, s \in S, n \in N \\
& w_{i} \in\{0,1\}, \quad \forall i \in I \\
& x_{i m} \geq 0 \text {, integer, } \forall i \in I, m \in M \\
& y_{i j m}^{s n} \geq 0 \text {, integer, } \\
& \forall i \in I, \quad j \in J, m \in M, s \in S, n \in N \\
& z_{i j k m}^{s n} \geq 0, \forall i \in I, j \in J, m \in M, k \in K, s \in S, n \in N .
\end{aligned}
$$

The proposed emergency supplies allocation model (50) for disaster response is a multiobjective mixed-integer nonlinear programming. The first nonlinear constraint with conditions (if) reflects that there exists the dependence between $\zeta_{i j}^{s n}$ and $\eta_{i j}^{s n}$. Binary variable $\zeta_{i j}^{s n}$ takes its value with 0 indicating that the path is destroyed. In this case, the available distance $\eta_{i j}^{s n}$ will equal an infinite number. In algorithm language, we can introduce a "big enough" constant, for example, 1000000. Similarly, the second nonlinear constraint with conditions (if) implies that the second-stage decision $y_{i j m}^{s n}$ is dependent on $\eta_{i j}^{s n}$. Once the available distance $\eta_{i j}^{s n}$ from relief supplier $i$ to affected area $j$ exceeds the threshold of coverage, let the decision variable $y_{i j m}^{s n}$ equal zero in the solution process. The multiple objectives structure of model (50) makes the use of goal programming appropriate particularly. This yields a rather efficient solution method for our model.

\section{Solution Method}

The deterministic equivalent formulation of original emergency logistics model is a multiobjective optimization problem. A great number of solution methods have been developed to solve multiobjective problems $[33,34]$. For example, Chang et al. [35] designed a greedy-search-based genetic algorithm that dynamically adjusted distribution schedules from various supply points according to the requirements at demand points to solve the relief allocation problem with three objectives. Huang et al. [36] adopted the weighted sum methods to transform a resource allocation and emergency distribution model with the humanitarian objectives, that is, lifesaving utility, delay cost, and fairness into a scalar optimization problem, which was solved by a variational inequality algorithm. Najafi et al. [37] proposed a hierarchical-objective-function-based solution methodology for a multiobjective, multicommodity, and multiperiod stochastic emergency logistics model.

In this paper, we adopt the goal programming method to integrate the two objectives and convert the multiobjective optimization problem to a single objective program. When planning the emergency logistics system, several humanitarian principles are presented in the original model, such as fairness and timeliness implications. Multiple goals with appropriate priority level must be taken into consideration, so it is a best choice to employ the goal programming method to formulate the hierarchical structure. The goal programming method was introduced by Charnes et al. [38]. Since it is easy to implement, the goal programming method has been applied in various fields. The solution process for our mixedinteger goal programming is described as follows.

Firstly, determine the optimization deviation function for every objective.

For the fairness objective function, the target value $G_{1}$ is given by the decision-maker who makes the decision relying on previous information or personal preference. When the target value is fixed, additional variables $d_{1}^{+}$and $d_{1}^{-}$can be obtained, serving as positive and negative deviation variables. The soft constraint is chalked up well by involving the negative and positive deviation variables together with the target value into the first objective. Note that the fairness objective is required to maximize. Therefore, the smaller $d_{1}^{+}$ is, the best the objective is:

$$
\begin{array}{ll}
\min & d_{1}^{+} \\
\operatorname{s.t.} & \frac{\sum_{s \in S} \sum_{n \in N} \sum_{j \in J} \sum_{k \in K} p_{s} q_{n}\left(d_{j k}^{s n}-\sum_{i \in I} \sum_{m \in M} z_{i j k m}^{s n}\right)}{\sum_{s \in S} \sum_{n \in N} \sum_{j \in J} \sum_{k \in K} p_{s} q_{n} d_{j k}^{s n}} \\
& +d_{1}^{-}-d_{1}^{+}=G_{1},
\end{array}
$$

where $d_{1}^{+}$represents the segment of the first objective exceeding its target value and $d_{1}^{-}$denotes the segment of the first objective less than its target value. 
Similarly, the timeliness objective function can be written as follows:

$$
\begin{array}{ll}
\min & d_{2}^{+} \\
\text {s.t. } & \sum_{s \in S} \sum_{n \in N} \sum_{i \in I} \sum_{j \in J} \sum_{m \in M} p_{s} q_{n} a_{m} \eta_{i j}^{s n} y_{i j m}^{s n}+d_{2}^{-}-d_{2}^{+} \\
& =G_{2},
\end{array}
$$

and the total cost objective function can be expressed as follows:

$$
\begin{array}{ll}
\min & d_{3}^{+} \\
\text {s.t. } \quad \sum_{i \in I} g_{i} w_{i}+\sum_{i \in I} \sum_{m \in M} c_{i m} x_{i m} \\
& +\sum_{s \in S} \sum_{n \in N} \sum_{i \in I} \sum_{j \in J} \sum_{m \in M} p_{s} q_{n} b_{m} \eta_{i j}^{s n} y_{i j m}^{s n}+d_{3}^{-}-d_{3}^{+} \\
& =G_{3} .
\end{array}
$$

Secondly, specify the preemptive level to construct the criteria function.

The decision-maker assigns these three objectives with preemptive levels $P_{1}, P_{2}$, and $P_{3}$, complying with $P_{1} \gg P_{2} \gg$ $P_{3}$. That is to say, when formulating the criteria function, it is requested to order the unfavorable deviations into a number of preemptive levels, with the minimization of a deviation in a higher preemptive level being infinitely more important than any deviations in lower preemptive levels. The criteria function minimizes the sum of the deviations from the target values specified in the soft constraints appended above; that is,

$$
\min P_{1} d_{1}^{+}+P_{2} d_{2}^{+}+P_{3} d_{3}^{+} \text {. }
$$

Thirdly, solve the deterministic mixed-integer goal programming model.

The preemptive goal programming formulation of emergency supplies allocation model is given as follows:

$$
\begin{array}{ll}
\min & P_{1} d_{1}^{+}+P_{2} d_{2}^{+}+P_{3} d_{3}^{+} \\
\text {s.t. } \quad \frac{\sum_{s \in S} \sum_{n \in N} \sum_{j \in J} \sum_{k \in K} p_{s} q_{n}\left(d_{j k}^{s n}-\sum_{i \in I} \sum_{m \in M} z_{i j k m}^{s n}\right)}{\sum_{s \in S} \sum_{n \in N} \sum_{j \in J} \sum_{k \in K} p_{s} q_{n} d_{j k}^{s n}} \\
\quad+d_{1}^{-}-d_{1}^{+}=G_{1} \\
\sum_{s \in S} \sum_{n \in N} \sum_{i \in I} \sum_{j \in J} \sum_{m \in M} p_{s} q_{n} a_{m} \eta_{i j}^{s n} y_{i j m}^{s n}+d_{2}^{-}-d_{2}^{+}=G_{2} \\
\sum_{i \in I} g_{i} w_{i}+\sum_{i \in I} \sum_{m \in M} c_{i m} x_{i m} \\
\quad+\sum_{s \in S} \sum_{n \in N} \sum_{i \in I} \sum_{j \in J} \sum_{m \in M} p_{s} q_{n} b_{m} \eta_{i j}^{s n} y_{i j m}^{s n}+d_{3}^{-}-d_{3}^{+}=G_{3} \\
x_{i m} \leq U_{i m} w_{i}, \quad \forall i \in I, m \in M \\
\sum_{j \in J} y_{i j m}^{s n} \leq x_{i m}, \quad \forall i \in I, m \in M, s \in S, n \in N
\end{array}
$$

$$
\sum_{k \in K} e_{k} z_{i j k m}^{s n} \leq V_{m} y_{i j m}^{s n}
$$

$\forall i \in I, j \in J, m \in M, s \in S, n \in N$

$$
\begin{aligned}
& \sum_{j \in J} \sum_{m \in M} z_{i j k m}^{s n} \leq \mu_{i k}+\sigma_{i k} \Phi^{-1}(1-\beta)-(2 \alpha-1) a_{i k}, \\
& \forall i \in I, k \in K, s \in S, n \in N \\
& \sum_{i \in I} \sum_{m \in M} z_{i j k m}^{s n} \leq d_{j k}^{s n}, \quad \forall j \in J, k \in K, s \in S, n \in N \\
& \eta_{i j}^{s n}=\left\{\begin{array}{ll}
t_{i j}, & \text { if } \zeta_{i j}^{s n}=1 \\
+\infty, & \text { if } \zeta_{i j}^{s n}=0,
\end{array} \quad \forall i \in I, j \in J, s \in S, n \in N\right. \\
& y_{i j m}^{s n} \begin{cases}\geq 0, & \text { if } \eta_{i j}^{s n} \leq T \\
=0, & \text { if } \eta_{i j}^{s n}>T,\end{cases}
\end{aligned}
$$$$
\forall i \in I, j \in J, m \in M, s \in S, n \in N
$$

$$
\begin{aligned}
& w_{i} \in\{0,1\}, \quad \forall i \in I \\
& x_{i m} \geq 0, \text { integer, } \quad \forall i \in I, m \in M \\
& y_{i j m}^{s n} \geq 0, \text { integer, }
\end{aligned}
$$$$
\forall i \in I, j \in J, m \in M, s \in S, n \in N
$$

$$
z_{i j k m}^{s n} \geq 0
$$$$
\forall i \in I, j \in J, m \in M, k \in K, s \in S, n \in N
$$

$d_{1}^{+}, d_{1}^{-}, d_{2}^{+}, d_{2}^{-}, d_{3}^{+}, d_{3}^{-} \geq 0$.

Model (55)-(70) is a deterministic mixed-integer parametric programming. Given the parameters $\alpha$ and $\beta$, we can make use of conventional optimization algorithms such as branch-and-bound method when the parameters vary in their domains. It is known that the Lingo is a state-of-the-art commercial tool including the branch-and-bound IP code. In the next section, the effectiveness of the goal programming method can be demonstrated via numerical experiments.

\section{Numerical Example}

5.1. Problem Description. Consider an illustrative example focusing on emergency supplies allocation for earthquake threats. There are four locations which may be selected as emergency facilities locations to provide emergency responses to cover four affected areas. Once a facility can be opened at any candidate location, the emergency resources stored in the response facilities contain three kinds of supplies: food, medical kits, and tent. These reliefs can be transported to the affected area by highway. Without loss of generality, multiple vehicles types are considered, such as light truck, medium truck, and heavy truck. The data about distance from the emergency facilities to the affected areas, vehicle scale, and cost in different emergency facilities are provided in Table 1 . The related parameters with respect to vehicles and emergency supplies are collected in Table 2. The main purpose of emergency response efforts is to provide shelter and assistance to disaster victims as 
TABLE 1: Parameters related to the distance, vehicle scale and cost.

\begin{tabular}{|c|c|c|c|c|c|c|c|c|c|c|c|}
\hline \multirow[t]{2}{*}{$i$} & \multirow{2}{*}{$g_{i}$} & \multicolumn{4}{|c|}{$\begin{array}{c}t_{i j} \\
j\end{array}$} & \multicolumn{3}{|c|}{$\begin{array}{c}U_{i m} \\
m\end{array}$} & \multicolumn{3}{|c|}{$\begin{array}{l}c_{i m} \\
m\end{array}$} \\
\hline & & 1 & 2 & 3 & 4 & 1 & 2 & 3 & 1 & 2 & 3 \\
\hline 1 & 150000 & 10,000 & 10,000 & 15,000 & 20,000 & 16 & 10 & 6 & 500 & 600 & 700 \\
\hline 2 & 130000 & 30,000 & 10,000 & 35,000 & 25,000 & 28 & 16 & 10 & 600 & 700 & 800 \\
\hline 3 & 140000 & 20,000 & 20,000 & 10,000 & 10,000 & 20 & 12 & 8 & 900 & 1000 & 1100 \\
\hline 4 & 120000 & 35,000 & 20,000 & 30,000 & 5,000 & 32 & 20 & 12 & 600 & 700 & 800 \\
\hline
\end{tabular}

TABLE 2: Other relevant parameters.

\begin{tabular}{ccccccc}
\hline & $m$ & $a_{m}$ & $b_{m}$ & $V_{m}$ & $k$ & $e_{k}$ \\
\hline 1 & Light truck & 0.06 & 0.1 & 5,000 & Food & 2 \\
2 & Medium truck & 0.07 & 0.2 & 10,000 & Medical kits & 5 \\
3 & Heavy truck & 0.09 & 0.3 & 20,000 & Tent & 100 \\
\hline
\end{tabular}

soon as possible. Under this consideration, suppose that the maximum coverage limit between the emergency facilities and affected areas is $30,000 \mathrm{~m}$.

Because of the inherent uncertainty of emergency logistics, the impact resulting from the extraordinary event, the supply amounts, demands of the affected areas, and availability of path are unknown prior to the event. Since supply is organized before the disaster occurs, it is appropriate to represent supply by fuzzy random variable with continuous possibility and probability distribution function in Table 3. On the other hand, the uncertainty of demand and availability of path is represented by a set of discrete scenarios with given probability and possibility of occurrence. There are four disaster scenarios $\left(\omega_{1}, \gamma_{1}\right),\left(\omega_{1}, \gamma_{2}\right),\left(\omega_{2}, \gamma_{1}\right)$, and $\left(\omega_{2}, \gamma_{2}\right)$, where the probabilities of $\omega_{1}$ and $\omega_{2}$ are $0.7,0.3$ and the possibilities of $\gamma_{1}$ and $\gamma_{2}$ are $1,0.75$, respectively. The detailed data related to demand and availability of path are displayed in Tables 4 and 5. In order to serve the faster delivery of supplies after the earthquake, our goal is to find an optimal strategy for allocating the emergency supplies in the emergency facilities and dispatching them to the affected areas.

5.2. Computational Results and Discussion. Suppose that $\alpha$ and $\beta$ equal 0.95 and 0.95 , respectively. We solve three single objective models by Lingo. The optimal values are $\min f_{1}=$ $0.098, \min f_{2}=0$, and $\min f_{3}=0$. Then, we set $G_{1}=0.098$, $G_{2}=0$, and $G_{3}=0$ in model (55). Taking use of goal programming method, the optimal value of first objective is 0.098, which implies that the goal of "fairness" is fully achieved. The optimal values of other two objectives are 51,626 s, and 219,431 RMB.

The optimum solutions under different scenarios are expressed in Figures 1-4. In every picture, the above row is on behalf of the emergency relief supplier, while the following row represents the affected area. The array of the line between the relief supplier and affected area is the integer numbers of light, medium, and heavy truck assigned to the path from emergency facility to affected area. From the computational results, we know that four candidate locations are opened as emergency facilities. The value of light truck, medium truck, and heavy truck in these four relief suppliers are $(10,16,6)$, $(0,0,8),(8,12,8)$, and $(1,0,3)$, respectively. On the other hand, these pictures also tell us the vehicle routing decisions under four different disaster scenarios. For instance, there are 8 light trucks, 11 medium trucks, and 1 heavy trucks assigned from the third relief supplier to the third affected area under mild pessimistic scenario. The value of the secondstage decision $z_{i j k m}^{s n}$ is omitted due to its huge number.

Next, we solve the single objective problem of $\max f_{1} \max f_{2}$ and $\max f_{3}$, respectively. The corresponding optimal values are $\max f_{1}=1, \max f_{2}=302,487.5$, and $\max f_{3}=780,287.5$. Based on the definition of objective achievement scale, we have

$$
\begin{aligned}
& \tau_{1}=\frac{\max f_{1}-\mathrm{obj}_{f_{1}}}{\max f_{1}-G_{1}}=100 \%, \\
& \tau_{2}=\frac{\max f_{2}-\mathrm{obj}_{f_{2}}}{\max f_{2}-G_{2}}=82.93 \%, \\
& \tau_{3}=\frac{\max f_{3}-\mathrm{obj}_{f_{3}}}{\max f_{3}-G_{3}}=71.88 \%,
\end{aligned}
$$

where $\mathrm{obj}_{f_{1}}, \mathrm{obj}_{f_{2}}$, and $\mathrm{obj}_{f_{3}}$ are the optimal values of three objectives by solving the equivalent goal programming model. Obviously, the "fairness" objective is achieved fully. At the same time, the timeliness objective and the economical efficiency are satisfied with the proportion more than $70 \%$.

\section{Conclusions}

The emergency supplies allocation plan can provide prompt and effective guidance for scientific decision-making and support in disaster management. In this paper, we studied the emergency supplies allocation problem under twofold uncertainty. We developed a novel equilibrium optimization method for emergency supplies allocation problem and built a kind of two-stage multiobjective optimization model, in which the demand, supply, and availability of the path were characterized by fuzzy random variables.

We addressed the case that the expected objectives and equilibrium constraints can be reduced to their equivalent forms. Theorem 2 showed that three objectives can be turned 
TABLE 3: The continuous distributions of supply amounts in different suppliers.

\begin{tabular}{cccc}
\hline$i$ & & $k$ & \\
& 1 & 2 & 3 \\
\hline 1 & $\left(X_{11, \omega}-2000, X_{11, \omega}, X_{11, \omega}+4000\right)$ & $\left(X_{12, \omega}-300, X_{12, \omega}, X_{12, \omega}+200\right)$ & $\left(X_{13, \omega}-50, X_{13, \omega}, X_{13, \omega}+40\right)$ \\
& $X_{11} \sim \mathcal{N}\left(42000,3000^{2}\right)$ & $X_{12} \sim \mathcal{N}\left(14000,1000^{2}\right)$ & $X_{13} \sim \mathcal{N}\left(1400,100^{2}\right)$ \\
\hline \multirow{2}{*}{2} & $\left(X_{21, \omega}-1000, X_{21, \omega}, X_{21, \omega}+3000\right)$ & $\left(X_{22, \omega}-300, X_{22, \omega}, X_{22, \omega}+500\right)$ & $\left(X_{23, \omega}-30, X_{23, \omega}, X_{23, \omega}+30\right)$ \\
& $X_{21} \sim \mathcal{N}\left(21000,1500^{2}\right)$ & $X_{22} \sim \mathcal{N}\left(7000,500^{2}\right)$ & $X_{23} \sim \mathcal{N}\left(700,50^{2}\right)$ \\
\hline \multirow{2}{*}{3} & $\left(X_{31, \omega}-3000, X_{31, \omega}, X_{31, \omega}+400\right)$ & $\left(X_{32, \omega}-1000, X_{32, \omega}, X_{32, \omega}+1200\right)$ & $\left(X_{33, \omega}-200, X_{33, \omega}, X_{33, \omega}+400\right)$ \\
& $X_{31} \sim \mathcal{N}\left(75600,5400^{2}\right)$ & $X_{32} \sim \mathcal{N}\left(25200,1800^{2}\right)$ & $X_{33} \sim \mathcal{N}\left(2520,180^{2}\right)$ \\
\hline \multirow{2}{*}{4} & $\left(X_{41, \omega}-200, X_{41, \omega}, X_{41, \omega}+400\right)$ & $\left(X_{42, \omega}-200, X_{42, \omega}, X_{42, \omega}+100\right)$ & $\left(X_{43, \omega}-20, X_{43, \omega}, X_{43, \omega}+30\right)$ \\
& $X_{41} \sim \mathcal{N}\left(8400,600^{2}\right)$ & $X_{42} \sim \mathcal{N}\left(2800,200^{2}\right)$ & $X_{43} \sim \mathcal{N}\left(280,20^{2}\right)$ \\
\hline
\end{tabular}

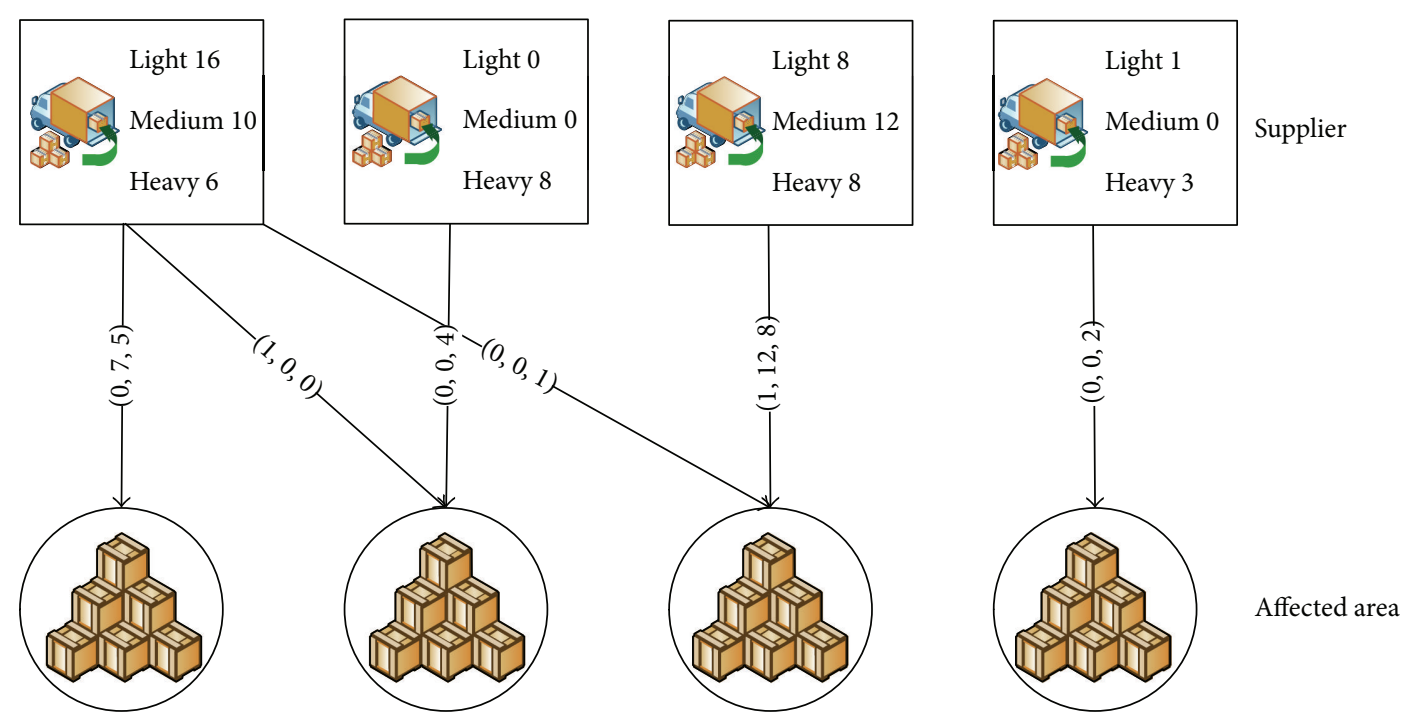

FIGURE 1: Decisions of supplier location and vehicle routing under mild optimistic scenario.

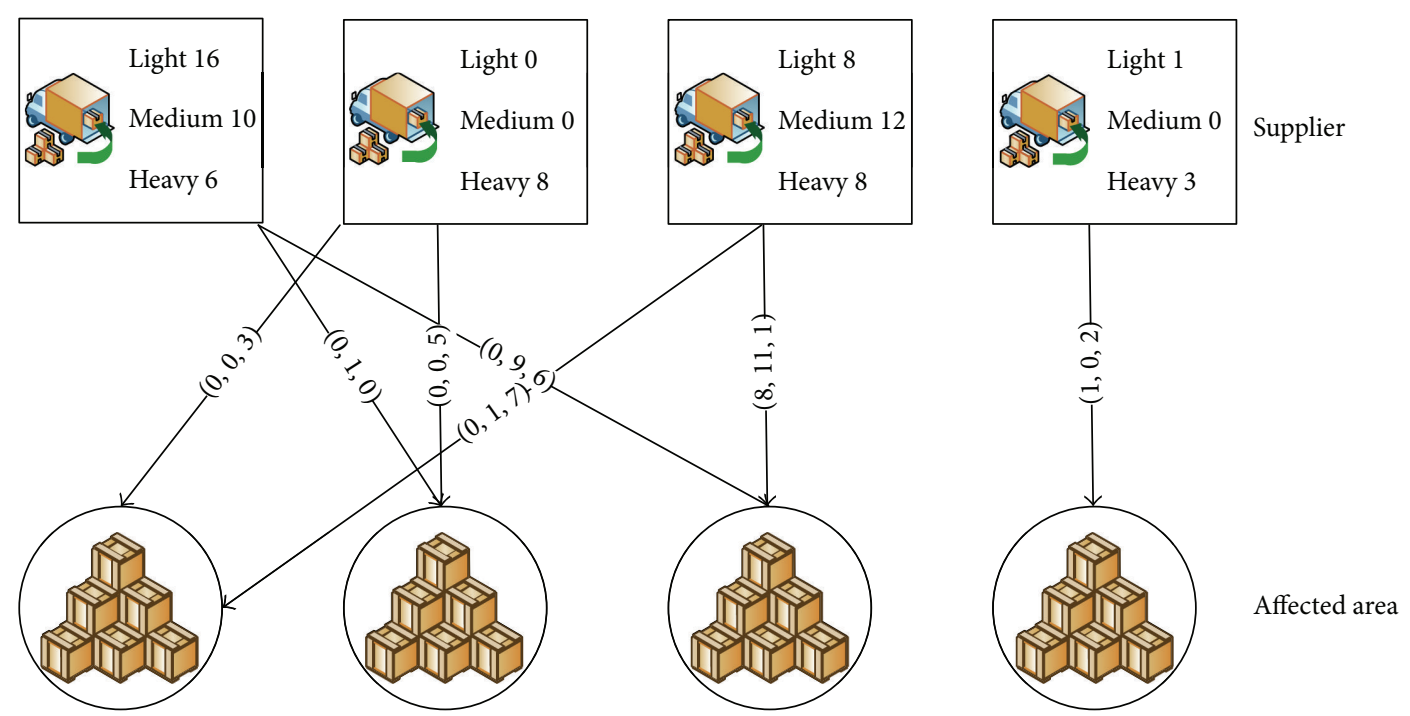

Figure 2: Decisions of supplier location and vehicle routing under mild pessimistic scenario. 


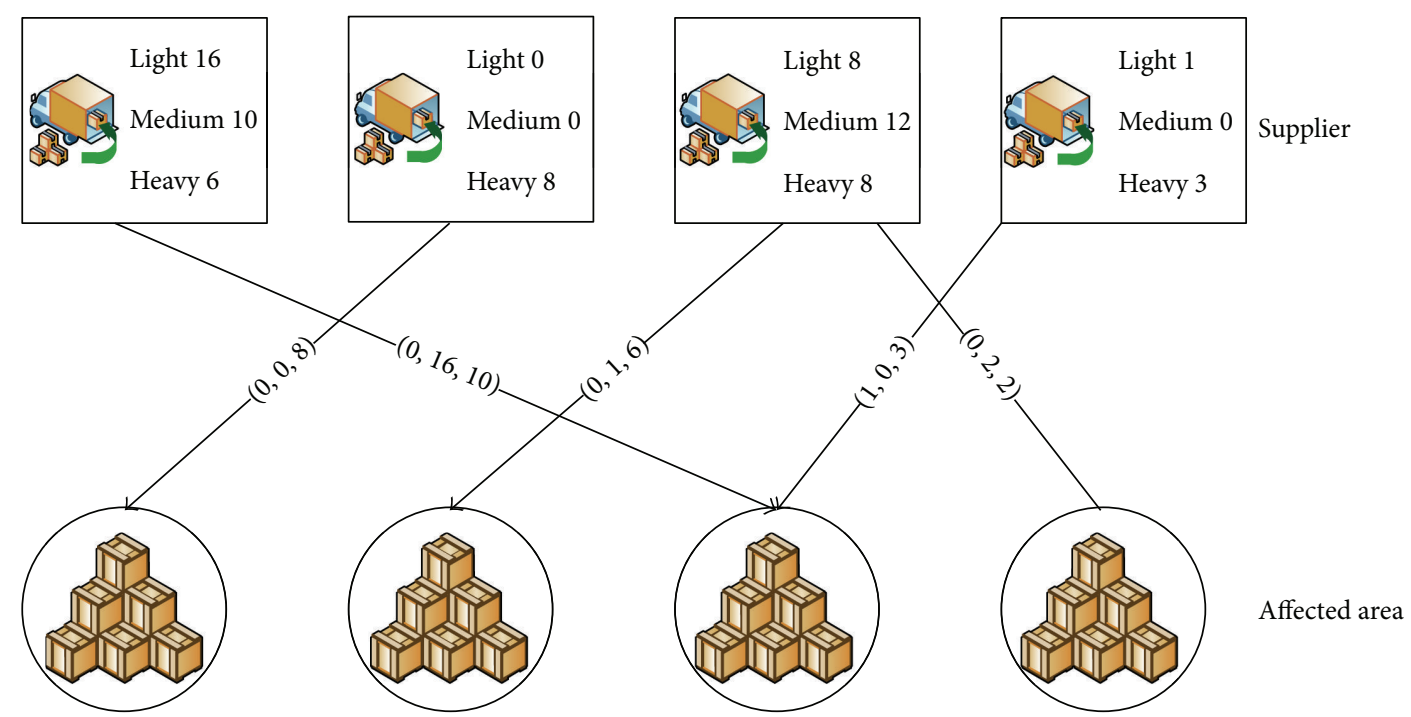

FIGURE 3: Decisions of supplier location and vehicle routing under severe optimistic scenario.

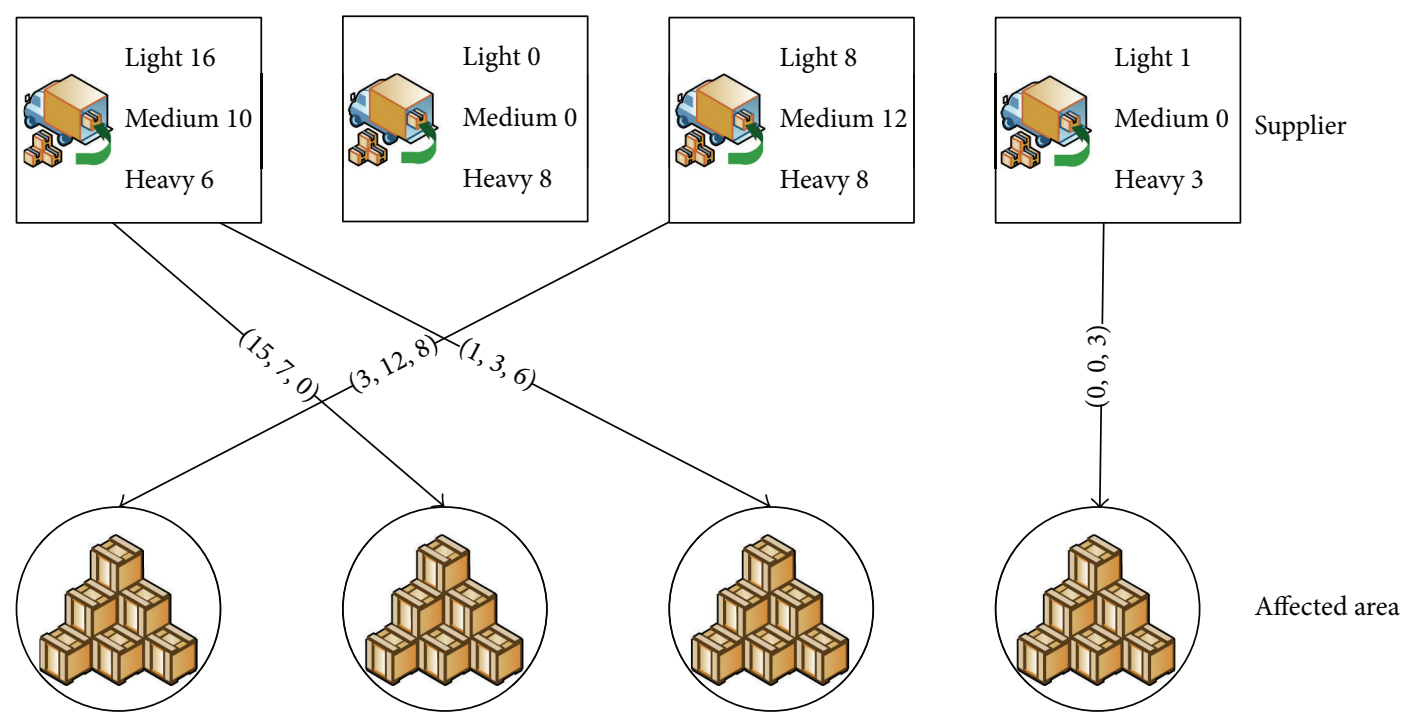

FIgURE 4: Decisions of supplier location and vehicle routing under severe pessimistic scenario.

to their equivalent ones in the case that the uncertain demand and availability of path were represented by a set of discrete disaster scenarios. Theorem 4 told us the equivalent expressions of equilibrium constraints when the fuzziness of uncertain supply followed triangular distribution and the randomness of uncertain supply obeyed normal distribution. Thus, we converted the proposed model into its multiobjective deterministic equivalent mixed-integer one.

Taking advantage of objective functions' preemptive level characteristics, we employed the goal programming method to solve the single objective version transformed. To test the performance of our equilibrium optimization and the effectiveness of the solution procedure, some illustrative experiments were conducted. To recognize the significant parameter of the proposed model, we also carried out the sensitivity analysis. The computational results showed that a decision-maker can make his scientific decisions by managing the equilibrium level according to his risk preference.

Our work focused on the emergency supplies allocation problem under demand and supply uncertainty with special distribution functions. The scope of future work could be extended to assume the uncertain parameter with general distributions and to explore more efficient customized algorithm designed for large-scale examples.

\section{Competing Interests}

The author declares that they have no competing interests. 
TABLE 4: The distributions of demands in different affected areas.

\begin{tabular}{|c|c|c|c|c|c|c|}
\hline \multirow{3}{*}{$j$} & \multicolumn{3}{|c|}{$d_{j k}^{11}$} & \multicolumn{3}{|c|}{$d_{j k}^{12}$} \\
\hline & \multicolumn{3}{|c|}{$k$} & \multicolumn{3}{|c|}{$k$} \\
\hline & 1 & 2 & 3 & 1 & 2 & 3 \\
\hline 1 & 24,000 & 8,000 & 800 & 30,000 & 10,000 & 1,000 \\
\hline 2 & 12,000 & 4,000 & 400 & 15,000 & 5,000 & 500 \\
\hline 3 & 43,200 & 14,400 & 1440 & 54,000 & 18,000 & 1,800 \\
\hline 4 & 4,800 & 1,600 & 160 & 6,000 & 2,000 & 200 \\
\hline \multirow{3}{*}{$j$} & \multicolumn{3}{|c|}{$d_{j k}^{21}$} & \multicolumn{3}{|c|}{$d_{j k}^{22}$} \\
\hline & \multicolumn{3}{|c|}{$k$} & \multicolumn{3}{|c|}{$k$} \\
\hline & 1 & 2 & 3 & 1 & 2 & 3 \\
\hline 1 & 36,000 & 12,000 & 1,200 & 42,000 & 14,000 & 1,400 \\
\hline 2 & 18,000 & 6,000 & 600 & 21,000 & 7,000 & 700 \\
\hline 3 & 64,800 & 21,600 & 2,160 & 75,600 & 25,200 & 2,520 \\
\hline 4 & 7,200 & 2,400 & 240 & 8,400 & 2,800 & 280 \\
\hline
\end{tabular}

TABLE 5: The availability between the suppliers and affected areas.

\begin{tabular}{|c|c|c|c|c|c|c|c|c|c|c|c|c|c|c|c|c|}
\hline \multirow{3}{*}{\multicolumn{2}{|c|}{1}} & & & & \multicolumn{4}{|c|}{$\zeta_{i j}^{12}$} & \multicolumn{4}{|c|}{$\zeta_{i j}^{21}$} & \multicolumn{4}{|c|}{$\zeta_{i j}^{22}$} \\
\hline & & & $j$ & & & & & & & & & & & & & \\
\hline & 1 & 2 & 3 & 4 & 1 & 2 & 3 & 4 & 1 & 2 & 3 & 4 & 1 & 2 & 3 & 4 \\
\hline 1 & 1 & 1 & 1 & 1 & 0 & 1 & 1 & 1 & 0 & 1 & 1 & 0 & 0 & 1 & 1 & 0 \\
\hline 2 & 1 & 1 & 1 & 1 & 1 & 1 & 1 & 1 & 1 & 0 & 1 & 0 & 0 & 0 & 1 & 0 \\
\hline 3 & 1 & 1 & 1 & 1 & 1 & 0 & 1 & 1 & 0 & 1 & 0 & 1 & 1 & 0 & 0 & 0 \\
\hline 4 & 1 & 1 & 1 & 1 & 1 & 1 & 0 & 1 & 1 & 0 & 1 & 1 & 1 & 0 & 0 & 1 \\
\hline
\end{tabular}

\section{Acknowledgments}

This work is supported partially by the Youth Natural Science Foundation of Hebei Province (no. A2016204057) and the National Natural Science Foundation of China (no. 61374184).

\section{References}

[1] N. Altay and W. G. Green III, "OR/MS research in disaster operations management," European Journal of Operational Research, vol. 175, no. 1, pp. 475-493, 2006.

[2] G. Galindo and R. Batta, "Review of recent developments in OR/MS research in disaster operations management," European Journal of Operational Research, vol. 230, no. 2, pp. 201-211, 2013.

[3] R. Abounacer, M. Rekik, and J. Renaud, "An exact solution approach for multi-objective location-transportation problem for disaster response," Computers \& Operations Research, vol. 41, no. 1, pp. 83-93, 2014.

[4] F. Barzinpour and V. Esmaeili, "A multi-objective relief chain location distribution model for urban disaster management," International Journal of Advanced Manufacturing Technology, vol. 70, no. 5, pp. 1291-1302, 2014.

[5] A. M. Anaya-Arenas, J. Renaud, and A. Ruiz, "Relief distribution networks: a systematic review," Annals of Operations Research, vol. 223, no. 1, pp. 53-79, 2014.

[6] M. C. Hoyos, R. S. Morales, and R. Akhavan-Tabatabaei, "OR models with stochastic components in disaster operations management: a literature survey," Computers \& Industrial Engineering, vol. 82, pp. 183-197, 2015.
[7] L. Özdamar and M. A. Ertem, "Models, solutions and enabling technologies in humanitarian logistics," European Journal of Operational Research, vol. 244, no. 1, pp. 55-65, 2015.

[8] Y.-J. Zheng, S.-Y. Chen, and H.-F. Ling, "Evolutionary optimization for disaster relief operations: a survey," Applied Soft Computing Journal, vol. 27, pp. 553-566, 2015.

[9] A. Bozorgi-Amiri, M. S. Jabalameli, and S. M. Mirzapour Ale-Hashem, "A multi-objective robust stochastic programming model for disaster relief logistics under uncertainty," OR Spectrum, vol. 35, no. 4, pp. 905-933, 2013.

[10] J. Salmerón and A. Apte, "Stochastic optimization for natural disaster asset prepositioning," Production and Operations Management, vol. 19, no. 5, pp. 561-574, 2010.

[11] C. G. Rawls and M. A. Turnquist, "Pre-positioning of emergency supplies for disaster response," Transportation Research Part B: Methodological, vol. 44, no. 4, pp. 521-534, 2010.

[12] B. Balcik and D. Ak, "Supplier selection for framework agreements in humanitarian relief," Production and Operations Management, vol. 23, no. 6, pp. 1028-1041, 2014.

[13] Z. Zhang and H. Jiang, "A robust counterpart approach to the biobjective emergency medical service design problem," Applied Mathematical Modelling, vol. 38, no. 3, pp. 1033-1040, 2014.

[14] J.-B. Sheu, "An emergency logistics distribution approach for quick response to urgent relief demand in disasters," Transportation Research Part E: Logistics and Transportation Review, vol. 43, no. 6, pp. 687-709, 2007.

[15] B. Sun, W. Ma, and H. Zhao, "A fuzzy rough set approach to emergency material demand prediction over two universes," Applied Mathematical Modelling, vol. 37, no. 10-11, pp. 70627070, 2013.

[16] Y.-J. Zheng and H.-F. Ling, "Emergency transportation planning in disaster relief supply chain management: a cooperative fuzzy optimization approach," Soft Computing, vol. 17, no. 7, pp. 1301-1314, 2013.

[17] X. Bai, "Optimization for pre-positioning emergency supplies problem under fuzzy environment," Systems EngineeringTheory \& Practice, vol. 35, no. 6, pp. 1465-1473, 2015.

[18] X. Bai and Y. K. Liu, "CVAR reduced fuzzy variables and their second order moments," Iranian Journal of Fuzzy Systems, vol. 12, no. 5, pp. 45-75, 2015.

[19] X. Bai and Y. K. Liu, "Robust optimization of supply chain network design in fuzzy decision system," Journal of Intelligent Manufacturing, 2014.

[20] H. Kwakernaak, "Fuzzy random variables I: definitions and theorems," Information Sciences, vol. 15, no. 1, pp. 1-29, 1978.

[21] Y.-K. Liu and B. Liu, "Fuzzy random variables: a scalar expected value operator," Fuzzy Optimization and Decision Making, vol. 2, no. 2, pp. 143-160, 2003.

[22] F. Ben Abdelaziz and H. Masri, "Multistage stochastic programming with fuzzy probability distribution," Fuzzy Sets and Systems, vol. 160, no. 22, pp. 3239-3249, 2009.

[23] S. Wang and J. Watada, "Two-stage fuzzy stochastic programming with Value-at-Risk criteria," Applied Soft Computing Journal, vol. 11, no. 1, pp. 1044-1056, 2011.

[24] S. Wang and W. Pedrycz, "Robust granular optimization: a structured approach for optimization under integrated uncertainty," IEEE Transactions on Fuzzy Systems, vol. 23, no. 5, pp. 1372-1386, 2015.

[25] Y. P. Li, G. H. Huang, Y. F. Huang, and H. D. Zhou, "A multistage fuzzy-stochastic programming model for supporting sustainable water-resources allocation and management," 
Environmental Modelling \& Software, vol. 24, no. 7, pp. 786-797, 2009.

[26] S. Wang, J. Watada, and W. Pedrycz, "Recourse-based facilitylocation problems in hybrid uncertain environment," IEEE Transactions on Systems, Man, and Cybernetics, Part B: Cybernetics, vol. 40, no. 4, pp. 1176-1187, 2010.

[27] S. Wang and J. Watada, "A hybrid modified PSO approach to VaR-based facility location problems with variable capacity in fuzzy random uncertainty," Information Sciences, vol. 192, no. 1, pp. 3-18, 2012.

[28] G. Yuan, "Two-stage fuzzy production planning expected value model and its approximation method," Applied Mathematical Modelling, vol. 36, no. 6, pp. 2429-2445, 2012.

[29] S. Wang, J. Watada, and W. Pedrycz, "Granular robust meanCVaR feedstock flow planning for waste-to-energy systems under integrated uncertainty," IEEE Transactions on Cybernetics, vol. 44, no. 10, pp. 1846-1857, 2014.

[30] Y.-K. Liu and B. Liu, "Fuzzy random programming with equilibrium chance constraints," Information Sciences, vol. 170, no. 2-4, pp. 363-395, 2005.

[31] H. Zhai, Y.-K. Liu, and K. Yang, "Modeling two-stage UHL problem with uncertain demands," Applied Mathematical Modelling, vol. 40, no. 4, pp. 3029-3048, 2016.

[32] Y. Wang, Y. Chen, and Y. Liu, "Modeling portfolio optimization problem by probability-credibility equilibrium risk criterion," Mathematical Problems in Engineering, vol. 2016, Article ID 9461021, 13 pages, 2016.

[33] A. Chinchuluun and P. M. Pardalos, "A survey of recent developments in multiobjective optimization," Annals of Operations Research, vol. 154, pp. 29-50, 2007.

[34] W.-M. Gai, Z.-A. Jiang, Y.-F. Deng, J. Li, and Y. Du, "Multiobjective route planning model and algorithm for emergency management," Mathematical Problems in Engineering, vol. 2015, Article ID 565403, 17 pages, 2015.

[35] F.-S. Chang, J.-S. Wu, C.-N. Lee, and H.-C. Shen, "Greedysearch-based multi-objective genetic algorithm for emergency logistics scheduling," Expert Systems with Applications, vol. 41, no. 6, pp. 2947-2956, 2014.

[36] K. Huang, Y. Jiang, Y. Yuan, and L. Zhao, "Modeling multiple humanitarian objectives in emergency response to large-scale disasters," Transportation Research Part E: Logistics and Transportation Review, vol. 75, pp. 1-17, 2015.

[37] M. Najafi, K. Eshghi, and W. Dullaert, "A multi-objective robust optimization model for logistics planning in the earthquake response phase," Transportation Research Part E: Logistics and Transportation Review, vol. 49, no. 1, pp. 217-249, 2013.

[38] A. Charnes, W. W. Cooper, and R. O. Ferguson, "Optimal estimation of executive compensation by linear programming," Management Science, vol. 1, pp. 138-151, 1955. 


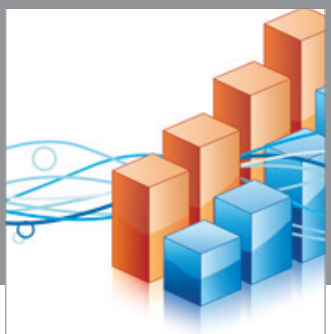

Advances in

Operations Research

vatem alat4

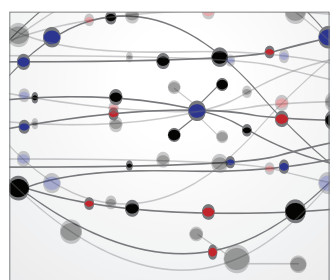

\section{The Scientific} World Journal
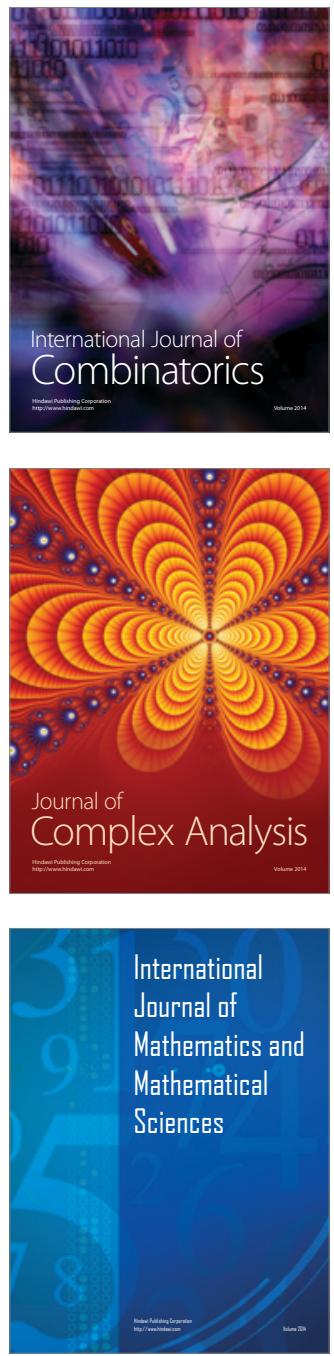
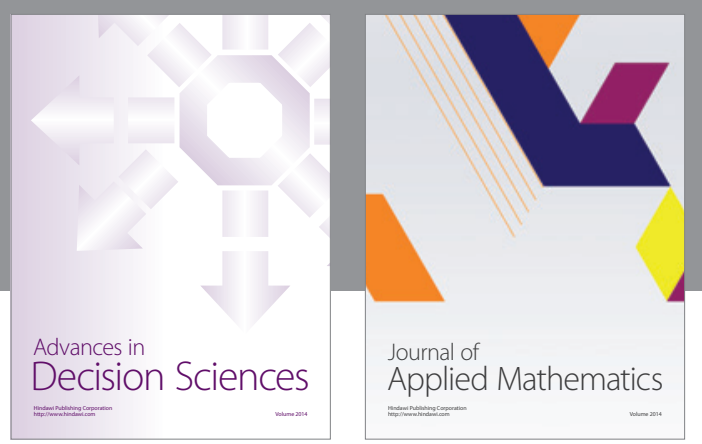

Algebra

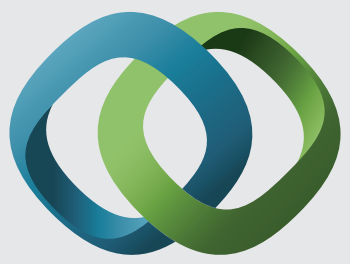

\section{Hindawi}

Submit your manuscripts at

http://www.hindawi.com
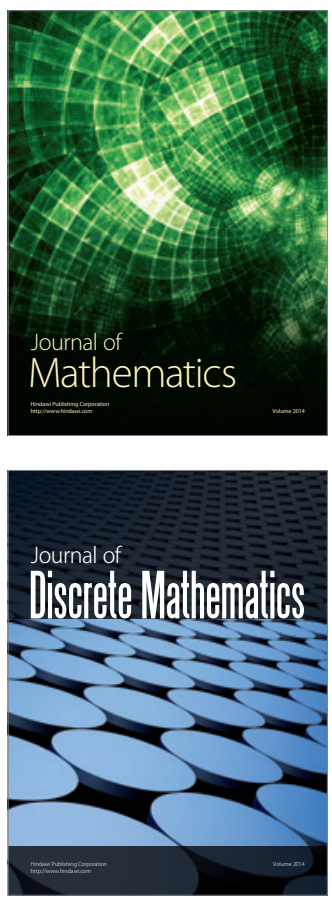

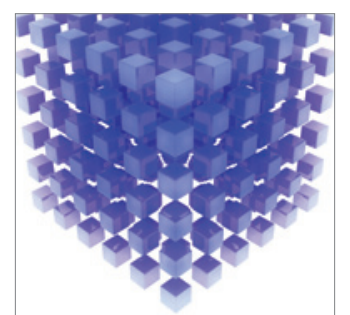

Mathematical Problems in Engineering
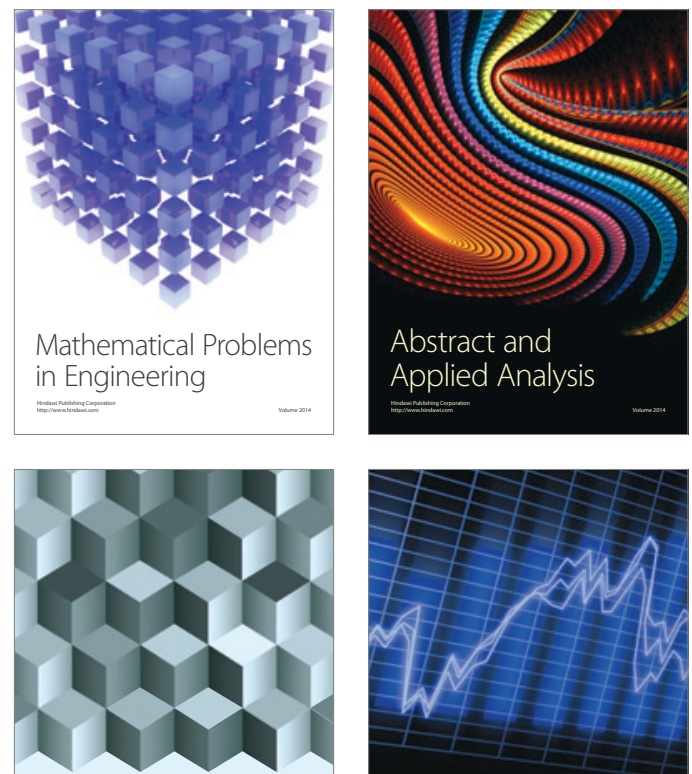

Journal of

Function Spaces

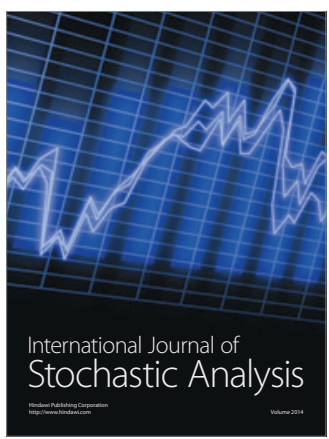

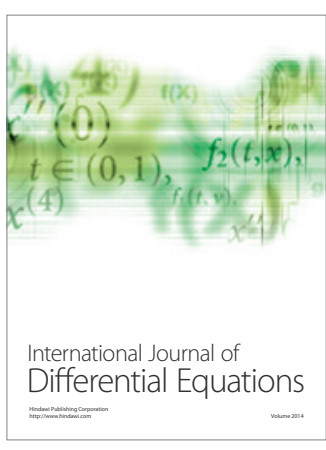
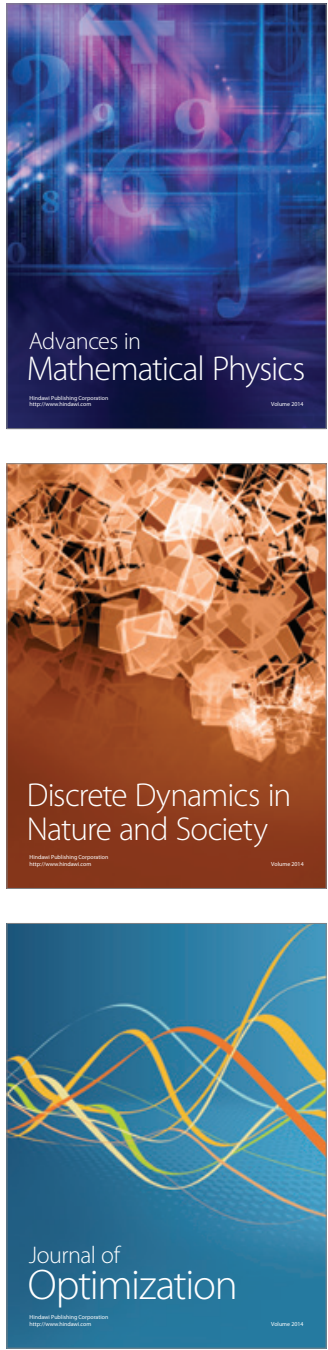